\title{
Publicação de trabalhos científicos apresentados em Congresso de Trauma no Brasil
}

\section{Publication of papers presented in a Brazilian Trauma Congress}

\author{
Vitor Augusto de Andrade 1; Stela Carpini 1; Ricardo Schwingel 1; Thiago Rodrigues Araújo Calderan 2 ; \\ Gustavo Pereira Fraga, TCBC-SP 3
}

\author{
R E S U M O
}

\begin{abstract}
Objetivo: Analisar a proporção de trabalhos apresentados no XXI Panamerican Congress of Trauma, VIII Congresso da Sociedade Brasileira de Atendimento Integrado ao Traumatizado (SBAIT) e X Congresso Brasileiro das Ligas do Trauma (CoLT) que foram publicados integralmente. Métodos: Nos eventos citados que foram sediados em Campinas, em 2008, foram apresentados e publicados os resumos de 347 trabalhos. Para avaliar a proporçãode trabalhos completos publicados foi realizado um estudo retrospectivo observacional consultando as bases de dados biomédicas PubMed e SciELO, com apoio do Google, a partir do título dos resumos e da listagem de autores. Resultados: Dos 347 trabalhos considerados, 25 (7,3\%) eram de serviços estrangeiros e $322(92,7 \%)$ de nacionais. Dez (2,9\%) trabalhos foram publicados, dos quais, seis $(1,7 \%)$ da área de enfermagem e quatro $(1,2 \%)$ de medicina. Dentre estes, foram identificados quatro publicações de tese e apenas um dos trabalhos internacionais foi publicado. Conclusão: Apesar de haver um grande número de trabalhos apresentados em congressos de trauma no Brasil, as publicações nesta área são raras. A academia e as sociedades de cirurgia precisam estimular a submissão de trabalhos científicos mesmo antes da apresentação em congressos a fim de serem avaliados para publicação em revistas indexadas.
\end{abstract}

Descritores: Ferimentos e lesões. Publicações científicas e técnicas. Eventos científicos e de divulgação.

\section{INTRODUÇÃO}

$M$ uitas faculdades, serviços e ligas acadêmicas no Brasil abordam a doença trauma em seus projetos de ensino e pesquisa. Por meio destes trabalhos científicos é possível promover o desenvolvimento e o progresso do conhecimento, propondo mudança nas formas de abordagem e intervenção às vítimas de traumas. A importância da pesquisa científica é um fato notadamente reconhecido e, no Brasil, a sua produção cresceu significativamente nos últimos anos, o que é confirmado pelo aumento do número de artigos de autores brasileiros publicados em periódicos qualificados pela indexação no Institute for Science Information ${ }^{1,2}$

A divulgação de trabalhos originais geralmente é feita por meio de publicações em periódicos especializados ou em eventos científicos, por meio de apresentações orais ou pôsteres. Nestes encontros são promovidos, além da imediata difusão do conhecimento, as discussões, o estímulo ao pensamento intelectual e a colaboração entre cientistas de todo mundo ${ }^{3}$. As apresentações de resumos sejam orais ou pôsteres, são considerados partes integrantes do intercambio de informações científica, sendo reconhecidas como parte do progresso científico ${ }^{4,5}$. Porém, uma vez não publicados, os trabalhos ficam restritos aos participantes destes encontros e limitando os avanços.

Em um estudo feito pela Cochrane Library, que revisou as taxas de publicações de resumos apresentados em encontros de diversas especialidades, encontrou apenas $45 \%$ publicados ${ }^{6}$. Na área trauma, apesar do aumento da produção cientifica pelos serviços de todo Brasil, o número de trabalhos, oriundos de autores brasileiros, encontrados em periódicos internacionais ou mesmo nacionais é muito baixo, mesmo sendo identificado, nas últimas décadas, um número crescente de eventos. Estes estudos, uma vez publicados e difundidos para toda sociedade podem ser úteis para o conhecimento geral, conscientização e planejamento, por parte de agências governamentais, de investimentos e programas na área de trauma.

Em 2008 foram realizados simultaneamente em Campinas o XXI Panamerican Congress of Trauma, orga-

Trabalho realizado na Disciplina de Cirurgia do Trauma do Departamento de Cirurgia da Faculdade de Ciências Médicas (FCM) da Universidade Estadual de Campinas (Unicamp) - SP-BR.

1. Acadêmico(a) do Curso de Medicina da FCM - Unicamp e membro da Liga do Trauma da Disciplina de Cirurgia do Trauma da FCM - UnicampCampinas - SP-BR; 2. Médico Residente da Disciplina de Cirurgia do Trauma da FCM - Unicamp- Unicamp- Campinas - SP-BR; 3. Professor Doutor e Coordenador da Disciplina de Cirurgia do Trauma da FCM - Unicamp - Unicamp- Campinas - SP-BR. 
nizado pela Sociedade Panamericana do Trauma (SPT), o VIII Congresso da SBAIT (Sociedade Brasileira de Atendimento Integrado ao Traumatizado) e o X Congresso Brasileiro das Ligas do Trauma (COLT). O evento reuniu durante cinco dias representantes de 21 países, trazendo entre os palestrantes, 68 médicos estrangeiros e 350 nacionais 7 . Durante o encontro, muitos trabalhos foram apresentados na forma de temas livres e pôsteres oriundos de profissionais acadêmicos de várias cidades do Brasil e outros países $^{8}$. Trabalhos como estes, apresentados em encontros científicos sobre o tema trauma são, muitas vezes, inacessíveis a outros profissionais por ficarem restritos à apresentação neste tipo de evento. A nossa hipótese é que a literatura em trauma ainda possui poucos trabalhos científicos originados de autores brasileiros e por isso este artigo tem a finalidade de analisar a proporção de trabalhos apresentados nestes três eventos e que foram publicados integralmente em periódicos.

\section{MÉTODOS}

Em 2008, no XXI Panamerican Congress of Trauma, no VIII Congresso da Sociedade Brasileira de Atendimento Integrado ao Traumatizado (SBAIT) e no X Congresso Brasileiro das Ligas do Trauma (CoLT), eventos simultâneos que ocorreram na cidade de Campinas, SP, entre os dias 19 e 22 de Novembro, foram apresentados 347 trabalhos, sendo 163 sob a forma de tema livre e 184 de pôster. Os resumos de todos os estudos, publicados nos anais do congresso e contidos na Revista do Colégio Brasileiro de Cirurgiões, volume 35, suplemento 1, 2008, foram analisados neste estudo.

Através dos títulos dos 347 resumos foi realizado um estudo retrospectivo observacional, até o mês de Agosto de 2010, consultando as principais bases de dados biomédicas, PubMed (http://www.ncbi.nlm.nih.gov/ pubmed/) e Scielo (http://www.scielo.org/php/index.php), com apoio do Google (http://www.google.com.br/). Para abranger os possíveis casos em que o título do resumo, ao ser publicado, foi alterado, portanto não encontrado nesta primeira busca, fez-se uma segunda pesquisa, tomando por base a listagem dos autores principais. Assim, para cada trabalho, foram realizadas duas consultas em cada banco de dados. Quando encontrado na íntegra, o resumo do estudo publicado passava por uma verificação, no intuito de conferir se era o mesmo estudo ou similar ao contido nos anais do congresso.

De todos os trabalhos foram coletados dados gerais (identificação do trabalho, categoria de apresentação, título inicial, nacionalidade e localização geográfica do serviço) e dados referentes aos que foram publicados (título publicado, periódico e local de indexação). Foi considerada a instituição do primeiro autor, sendo classificada como indeterminada quando os dados no resumo não permitiram identificar o serviço de origem. Ao final da consul- ta, todos os dados foram tabulados em planilha Excel $\circledast$ para análise e interpretação.

\section{RESULTADOS}

Foram apresentados 347 trabalhos (163 temas livres e 184 pôsteres), dos quais 25 (7,3\%) foram oriundos de serviços estrangeiros, sendo seis $(1,7 \%)$ dos EUA, seis $(1,7 \%)$ da Colômbia, três $(0,9 \%)$ do Canadá, três $(0,9 \%)$ do Chile, três $(0,9 \%)$ da Venezuela, e quatro de outros países (Tabela 1). Os outros 322 (92,7\%) trabalhos foram de autores nacionais, sendo que a região Sudeste respondeu por aproximadamente $50 \%$ da origem dos trabalhos, liderado por São Paulo, com 99 (28,5\%), seguido por Minas Gerais com 57 (16,4\%) (Tabela 2).

Avaliando os trabalhos apresentados, observouse que $49(14,1 \%)$ eram da área de enfermagem ou fisioterapia. Dez trabalhos (2,9\%) foram publicados, dos quais, seis $(1,7 \%)$ eram da área de enfermagem e quatro $(1,2 \%)$ de medicina. Portanto, foram publicados 1,3\% dos trabaIhos da área médica ou de ligas do trauma e 12,2\% dos trabalhos da áerea de enfermagem ou fisioterapia $(p<0.01)$. Dentre estes dez estudos, quatro $(1,2 \%)$ foram publicações de tese, sendo uma delas também publicada em revista internacional indexada no PubMed. Os outros seis trabalhos, uma publicação $(0,3 \%)$ foi encontrada em revista indexada no SciELO e duas $(0,6 \%)$ em revista indexada no PubMed e SciELO. Apenas um (0,3\%) dos trabalhos de autores internacionais foi publicado e em revista não indexada. Ainda pelo Google foram identificados outros dois $(0,6 \%)$ trabalhos nacionais publicados em revistas não indexadas (Tabela 3).

\section{DISCUSSÃO}

Os resultados encontrados mostraram um baixo índice de publicação, muito inferior as taxas de congressos internacionais, que chega a variar de 21 a $60 \%$ de resumos publicados ${ }^{9-12}$. Mesmo considerando as afirmações de

Tabela 1 - Classificação das apresentações segundo o país.

\begin{tabular}{lcc}
\hline País & N & (\%) \\
\hline Estados Unidos & 6 & 1,7 \\
Colômbia & 6 & 1,7 \\
Canadá & 3 & 0,9 \\
Chile & 3 & 0,9 \\
Venezuela & 3 & 0,9 \\
Argentina & 1 & 0,3 \\
Catar & 1 & 0,3 \\
Espanha & 1 & 0,3 \\
México & 1 & 0,3 \\
Total & 25 & $7,3 \%$ \\
\hline
\end{tabular}


Miguel-Dasit et al. ${ }^{11}$, de que o país de origem do trabalho e a colaboração internacional influenciam as probabilidades de que um trabalho venha a ser publicado, e as de von Elm et al. ${ }^{12}$, de que a taxa de publicação varia conforme a especialidade médica - congressos de pediatria e cirurgia teriam maiores taxas de publicação posterior de trabalhos completos do que congressos de anestesia e emergência, por exemplo, os números encontrados em nosso estudo parecem muito baixos.

Analisando-se resumos apresentados em um Congresso Brasileiro de Urologia em 2003, encontrou-se uma taxa de publicação de 39\%, comparável a congressos estrangeiros ${ }^{5}$, ao analisar-se os resumos de um congresso na área da pesquisa odontológica no interior de São Paulo foi encontrada uma taxa de $15 \%^{13}$ e uma taxa média de $6,3 \%$ de publicação em dois congressos de angiologia e cirurgia vascular brasileiros, revelando o quanto a publicação das pesquisas está relegada a segundo plano no Brasil ${ }^{14}$. Já analisando o índice de publicação de trabaIhos apresentados no XXIV Congresso Brasileiro de Cirurgia, encontrou-se uma taxa de $2,6 \%$, muito próxima da

Tabela 2 - Classificação das apresentações segundo o estado brasileiro.

\begin{tabular}{lrr}
\hline Estado & N & (\%) \\
\hline São Paulo & 99 & 28,5 \\
Minas Gerais & 57 & 16,4 \\
Paraná & 35 & 10,0 \\
Maranhão & 24 & 6,9 \\
Bahia & 24 & 6,9 \\
Rio Grande do Sul & 21 & 6,1 \\
Rio de Janeiro & 16 & 4,6 \\
Ceará & 11 & 3,2 \\
Paraíba & 6 & 1,7 \\
Sergipe & 5 & 1,4 \\
Roraima & 5 & 1,4 \\
Pernambuco & 5 & 1,4 \\
Amazonas & 4 & 1,2 \\
Distrito Federal & 3 & 0,9 \\
Espírito Santo & 2 & 0,6 \\
Pará & 2 & 0,6 \\
Goiás & 1 & 0,3 \\
Santa Catarina & 1 & 0,3 \\
Indefinido & 1 & 0,3 \\
Total & 322 & 92,7 \\
\hline
\end{tabular}

encontrada neste estudo, o que demonstra e confirma os baixos índices nacionais em determinados encontros ${ }^{10}$. Explicações para estes baixos números podem residir em possíveis falhas na opção metodológica, assim como em publicação com títulos diferentes dos trabalhos anteriormente apresentados nos encontros e por variações nos nomes dos autores principais, aqueles cujos nomes são os primeiros apresentados na citação. Além disso, alguns dos trabaIhos analisados no presente estudo ainda poderão ser publicados, ou estarem em fase de análise pelos periódicos, embora estimativas mostrem que a publicação ocorra dentro de até dois anos, na maioria dos casos ${ }^{9,12,15}$. Essa afirmação baseia-se, principalmente, nos dados de uma pesquisa que analisa 19.123 trabalhos apresentados em 234 encontros de 1957 a 1999 e que encontrou uma taxa de $27 \%$ de publicação depois de 2 anos e de $47 \%$ após seis anos.

Outra possibilidade para a não publicação é a rejeição dos manuscritos por uma exigência de rigor científico por parte dos periódicos, que seria maior se comparada à exigência para apresentação em congressos, pois resumos apresentados em encontros teriam a função de apresentação inicial de projetos de pesquisa finalizados, de estímulo para o seguimento dos estudos e trariam informações a um público restrito de participantes do evento ${ }^{13}$. É conhecido que o índice de rejeição pelos periódicos é grande, superando muitas vezes a marca dos $50 \%$. Em revistas de prestígio em enfermagem, por exemplo, o índice de aceitação é extremamente baixo, variando de 10 a 35\% em relação ao número submissões ${ }^{16,17}$. As razões para este fato são basicamente relacionadas ou a aspectos relativos à qualidade do trabalho em relação à revista selecionada, como fragilidades metodológicas, má apresentação e falta de discussão dos resultados, ou a aspectos técnicos do próprio preparo do artigo, como falta de equilíbrio entre as partes, poucas citações e textos muito curtos ou muito longos $^{18}$. Por outro lado, o tempo necessário para a redação de um resumo é significativamente menor do que para a redação do artigo em sua versão completa. Isso é relevante, considerando-se que uma importante razão para a não publicação alegada pelos pesquisadores é a falta de tempo ${ }^{18,19}$.

Outros fatores que influenciariam o filtro no caminho entre a apresentação de um trabalho em um congresso e sua posterior publicação identificados por von Elm et al. ${ }^{12}$ e que merecem destaque em nossa discussão seriam o viés de publicação, a área de pesquisa e o tamanho do congresso em que o trabalho foi apresentado. Segundo

Tabela 3 - Perfil das publicações.

\begin{tabular}{lccccc}
\hline Area & Publicações & Teses & PubMed & SciELO & Revistas não indexadas \\
\hline Enfermagem & $6 / 49(1,7 \%$ do total $)$ & 3 & 1 & 2 & 1 \\
Medicina & $4 / 298(1,2 \%$ do total $)$ & 1 & 2 & 1 & 2 \\
Total & $10(2,9 \%)$ & 4 & 3 & 3 & 3 \\
\hline
\end{tabular}


esse e outros autores, pesquisas com resultados positivos teriam maiores chances de publicação subseqüente do que pesquisas com resultados negativos (viés de publicação de desfecho positivo ${ }^{12,19}$. Trabalhos na área da pesquisa básica também teriam maior probabilidade de serem publicados. O mesmo ocorreria com trabalhos apresentados em congressos menores, por terem uma seleção mais rigorosa do que os grandes encontros, em que os organizadores, para atraírem um maior público, aceitariam trabalhos com menor exigência, levando à seleção de trabalhos de menor qualidade. Talvez nossos baixos números tenham sido influenciados pelo fato de o congresso aqui analisado ter sido um evento grande e ter reunido simultaneamente três encontros (o XXI Panamerican Congress of Trauma, o VIII Congresso da SBAIT e o X Congresso Brasileiro das Ligas do Trauma). Porém, chamou a atenção a maior taxa de publicação de trabalhos de enfermagem e fisioterapia se comparado a medicina e ligas do trauma (12,2 vs. 1,3\%). Talvez isso tenha sido decorrente do estímulo nas faculdades e serviços principalmente de enfermagem para apresentação de trabalhos de qualidade em um congresso de tradição internacional realizado no Brasil.

Também foi observada a elevada proporção de trabalhos provenientes das regiões sul e sudeste, em que São Paulo sozinho corresponde por $28,5 \%$ dos estudos. Esta disparidade de produtividade científica relacionada à procedência por regiões brasileiras foi encontrada também em outras especialidades médicas por outros autores, pa- rece ser um reflexo da elevada concentração de universidades e centros de estudos na região, com a atração de elevados investimentos e incentivos, haja vista que esta é a região mais industrializada e desenvolvida do país ${ }^{20-22}$.

Algo que pode estar associado a essa baixa produção de artigos de trauma no Brasil é que ainda não existe a organização de sistemas, centros e registros de trauma em nosso país. São poucos os serviços que organizam periodicamente reuniões de morbidade e mortalidade, avaliação dos casos de óbito evitável e o desenvolvimento do controle de qualidade, ficando evidente a necessidade de uma maior atenção à doença trauma em nosso meio ${ }^{23}$.

Mesmo havendo um elevado número de trabaIhos apresentados nesses congressos de trauma no Brasil, as publicações foram raras e, quando realizadas, oriundas de grupos de poucos centros. Destas, três foram na forma de teses, as quais tratam-se de estudos reflexivos-teóricos, com apresentação de idéias do autor, e portanto, apresentam conteúdo limitado, questionando-se assim até mesmo a validade da publicação enquanto objeto de divulgação de novas informações para a comunidade científicas.

Os resultados encontrados sugerem a necessidade de medidas para o incremento dos índices de publicação. Uma sugestão, a exemplo de congressos internacionais, seria a obrigatoriedade de submissão do manuscrito à publicação anteriormente à apresentação em congressos, incentivando assim a disseminação da produção científica em trauma pelo país.

\section{A B S S T R A C T}

Objective: to assess the proportion of papers presented at the XXI Panamerican Congress of Trauma, VIII Congress of the Brazilian Society of Integrated Assistance to the Traumatized (SBAIT) and X Brazilian Congress of Trauma Leagues (CoLT) that were published in full. Methods: In the events cited, based in Campinas in 2008, 347 papers abstracts of were presented and published. To evaluate the proportion of complete published works a retrospective observational study was conducted, reviewing the biomedical databases PubMed and SCIELO, with support from Google, from the title of the abstracts and list of authors. Results: Of 347 papers considered, 25 (7.3\%) were from foreign services and 322 (92.7\%) national. Ten (2.9\%) papers were published, of which six (1.7\%) of nursing and four (1.2\%) medical. Among these, four thesis publications were identified and only one international work has been published. Conclusion: Despite a large number of papers presented at trauma conferences in Brazil, the publications in this area are rare. The academy and the societies of surgery need to encourage the submission of scientific papers even before the presentation in Congresses in order for them to be evaluated for publication in indexed journals.

Key words: Wounds and injuries. Scientific and technical publications. Scientific and educational events.

\section{REFERENCIAS}

1. Guimarães JA. A pesquisa médica e biomédica no Brasil: comparações com o desempenho científico brasileiro e mundial. Ciênc saúde coletiva 2004; 9(2):303-27.

2. Fabro AT, Yoo HHB, Queluz THAT. Perfil da atividade de pesquisa publicada nos anais dos congressos brasileiros de pneumologia e tisiologia nos últimos vinte anos. J bras pneumol 2006; 32(4):30915

3. Falagas ME, Rosmarakis ES. Clinical decision-making based on findings presented in conference abstracts: is it safe for our patients ? Eur Heart J 2006; 27(17):2038-9
4. Byerly WG, Rheney CC, Connelly JF, Verzino KC. Publication rates of abstracts from two pharmacy meetings. Ann Pharmacother 2000; 34(10):1123-7.

5. Oliveira LR, Figueiredo AA, Choi M, Ferrarez CE, Bastos AN, Netto JM. The publication rate of abstracts presented at the 2003 urological Brazilian meeting. Clinics 2009; 64(4):345-9.

6. Cartwright $\mathrm{R}$, Khoo AK, Cardozo L. Publish or be damned ? The fate of abstracts presented at the International Continence Society Meeting 2003. Neurourol Urodyn 2007; 26(2):154-7.

7. Mantovani M. Trauma sem fronteiras. Rev Col Bras Cir 2008 35(5):282-3.

8. Temas Livres e Pôsteres inscritos no XXI Panamerican Congress of Trauma, VIII Congresso da Sociedade Brasileira de Atendimento 
Integrado ao Traumatizado (SBAIT) e X Congresso Brasileiro das Ligas do Trauma (CoLT). Rev Col Bras Cir 2008; 35(Suppl 1).

9. Marx WF, Cloft HJ, Do HM, Kallmes DF. The fate of neuroradiologic abstracts presented at national meetings in 1993: rate of subsequent publication in peer-reviewed, indexed journals. AJNR Am J Neuroradiol 1999; 20(6):1173-7.

10. Fernandes FAMH, Ventura DE, Del Grande JC. Índice de publicação dos trabalhos apresentados no XXIV Congresso Brasileiro de Cirurgia. Rev Col Bras Cir 2003; 30(5):392-5.

11. Miguel-Dasit A, Martí-Bonmatí L, Aleixandre R, Sanfeliu P, Bautista $D$. Publication of material presented at radiologic meetings: authors2 country and international collaboration. Radiology 2006; 239(2):521-8.

12. von Elm E, Costanza MC, Walder $B$, Tramèr MR. More insight into the fate of biomedical meeting abstracts: a systematic review. BMC Med Res Methodol 2003; 3:12.

13. Leles CR, Rocha SS, Simões PA, Compagnoni MA. Taxa de publicação, na forma de artigos completos, de resumos apresentados em evento científico de pesquisa odontológica. Rev odontol UNESP 2006: 35(3):141-7.

14. Yoshida WB, Holmo NF, Corregliano GT, Baldon KM, Silva NS. Publicações indexadas geradas a partir de resumos de congressos de angiologia e cirurgia vascular no Brasil. J vasc bras 2008; 7(4):2937.

15. Scherer RW, Dickersin K, Langenberg P. Full publication of results initially presented in abstracts. A meta-analysis. JAMA 1994; 272(2):158-62

16. Henry B. Writing for scientific publication. Acta Paul Enf 2002; 15(1):59-71.

17. Silva MJP, Egry EY, Ângelo M, Barbosa MAM, Sousa RMC, Castilho $V$, et al. Produção do conhecimento em Enfermagem: da idéia da pesquisa à publicação em periódico qualificado. Rev esc enferm USP 2009; 43(2):1347-51.
18. Sprague S, Bhandari M, Devereaux PJ, Swiontkowski MF, Tornetta P 3rd, Cook DJ, et al. Barriers to full-text publication following presentation of abstracts at annual orthopaedic meetings. J Bone Joint Surg Am 2003; 85-A(1):158-63.

19. Stern JM, Simes RJ. Publication bias: evidence of delayed publication in a cohort study of clinical research projects. BMJ 1997; 315(7109):640-5.

20. Gomes SP, Santos MALC. Avaliação de um periódico na área de medicina tropical. Ci Inf 2001; 30(2):91-100.

21. Galileu D, Rocha FF, Nicolato R, Teixiera AL, Romano-Silva MA, Correa $\mathrm{H}$. Produção brasileira em periódicos psquiátricos de alto fator de impacto em 2005. J bras psiquiatr 2006; 55(2):120-4.

22. Freitas CM. A produção científica sobre o ambiente na saúde coletiva. Cad Saúde Pública 2005; 21(3):679-701.

23. Fraga GP. Programas de qualidade no atendimento ao trauma. Medicina (Ribeirão Preto) 2007; 40(3):321-8.

Recebido em 03/05/2010

Aceito para publicação em 06/07/2010

Conflito de interesse: nenhum

Fonte de financiamento: O XXI Panamerican Congress of Trauma, VIII Congresso da Sociedade Brasileira de Atendimento Integrado ao Traumatizado (SBAIT) e X Congresso Brasileiro das Ligas do Trauma (COLT) receberam apoio financeiro da FAPESP e CAPES para a sua realização.

\section{Como citar este artigo:}

Andrade VA, Carpini S, Schwingel R, Calderan Fraga GP. Publicação de trabalhos científicos apresentados em congressos de trauma no Brasil. Rev Col Bras Cir. [periódico na Internet] 2011; 38(3). Disponível em URL: http://www.scielo.br/rcbc

Endereço para correspondência:

Gustavo Pereira Fraga

E-mail: fragagp2008@gmail.com 